INPLASY

PROTOCOL

To cite: Zhou et al. Sleep duration and the risk of major eye diseases: A systematic review and meta-analysis. Inplasy protocol 202190046. doi:

10.37766/inplasy2021.9.0046

Received: 16 September 2021

Published: 16 September 2021

Corresponding author:

Chen-Wei Pan

15062317163@163.com

Author Affiliation:

School of Public Health, Medical College of Soochow University, Suzhou, China.

Support: NSFC no. 82122059.

Review Stage at time of this submission: Piloting of the study selection process.

Conflicts of interest:

None declared.

\section{Sleep duration and the risk of major eye diseases: A systematic review and meta-analysis}

Zhou, M1; Kai, JY2; Pan, CW³.

Review question / Objective: We conducted this systematic review and meta-analysis to assess the relationship between sleep duration and the risk of major eye diseases such as myopia, glaucoma, cataract, age-related macular degeneration (AMD), and diabetic retinopathy (DR).

Condition being studied: The evidence for a link between sleep duration and major eye diseases is accumulating, whereas the available literature has not yet been systematically reviewed.

Information sources: The pertinent literature was sourced using PubMed, Embase, Web of Science, and Cochrane library before July 2021. The search keywords were presented as follows: "sleep" as exposure factors; and "myopia" or "glaucoma" or "cataract" or "age-related macular degeneration" or "diabetic retinopathy", as outcome factors. Additionally, we also searched reference lists of identified studies to avoid omitting potentially relevant studies.

INPLASY registration number: This protocol was registered with the International Platform of Registered Systematic Review and Meta-Analysis Protocols (INPLASY) on 16 September 2021 and was last updated on 16 September 2021 (registration number INPLASY202190046).

\section{INTRODUCTION}

Review question / Objective: We conducted this systematic review and meta-analysis to assess the relationship between sleep duration and the risk of major eye diseases such as myopia, glaucoma, cataract, agerelated macular degeneration (AMD), and diabetic retinopathy (DR).

Condition being studied: The evidence for a link between sleep duration and major eye 
diseases is accumulating, whereas the available literature has not yet been systematically reviewed.

\section{METHODS}

Search strategy: In this review, databases including PubMed, Embase, Web of Science, and Cochrane Library were searched for eligible publications before July 2021. Full search terms used for PubMed were presented as follows: "sleep"[MeSH Terms] AND ("myopia"[Title/ Abstract] OR "glaucoma"[Title/Abstract] OR "cataract"[Title/Abstract] OR "diabetic retinopathy"[Title/Abstract] OR "agerelated macular degeneration"[Title/ Abstract] OR "age-related macular degeneration"[Title/Abstract] OR "agerelated maculopathy"[Title/Abstract] OR "age-related maculopathy"[Title/Abstract] OR "macular degeneration"[Title/ Abstract]).

Participant or population: Observational studies that enrolled participants of any age, healthy or diagnosed with any kind of major eye diseases will be included. Studies including participants with other diagnoses will be excluded.

Intervention: Long sleep duration or short sleep duration. Due to the between-study heterogeneity across studies, we will focus on the relative sleep duration instead of the absolute sleep duration in each study, and present the cut-points in sleep categories defined in the study.

Comparator: We will include studies where sleep duration is the only variation between the exposed group and the control group.

Study designs to be included: The study used a cross-sectional, case-control, or cohort design.

Eligibility criteria: Studies will be included if they: (1) were original studies; (2) regarded sleep duration as the exposure; (3) demonstrated the relationship between sleep duration and any one of major eye diseases; (4) provided sufficient data for the calculation of odds ratio (OR) and its $95 \%$ confidence interval (95\% Cl); (5) were published in English language.

Information sources: The pertinent literature was sourced using PubMed, Embase, Web of Science, and Cochrane library before July 2021. The search keywords were presented as follows: "sleep" as exposure factors; and "myopia" or "glaucoma" or "cataract" or "agerelated macular degeneration" or "diabetic retinopathy", as outcome factors. Additionally, we also searched reference lists of identified studies to avoid omitting potentially relevant studies.

Main outcome(s): We aim to systematically review the published literature on the relationship between sleep duration and the following major eye diseases: myopia, glaucoma, cataract, AMD, and DR. Odds ratios will be the effect measures for our main outcome.

Quality assessment / Risk of bias analysis: The quality of the eligible cross-sectional studies will be assessed by the Agency for Healthcare Research and Quality (AHRQ), whereas the quality of each included casecontrol and cohort study will be assessed using the Newcastle-Ottawa Scale (NOS). Two investigators will rate each study based on the relevant quality criteria independently, and any disagreement will be discussed and ultimately resolved by consensus.

Strategy of data synthesis: The ORs and 95\% Cls within each included study assessing the association between sleep duration and major eye diseases will be coded and fully combined to obtain a pooled OR and its corresponding $95 \% \mathrm{CI}$ using a random-effects model. When multiple models are used to adjust the OR value, the model with the most covariates included will be employed. The extent of heterogeneity among the available studies will be quantified using the $I^{2}$ statistical test. Evidence of publication bias will be assessed using the Egger's test, and the $p$ value will be less than 0.05 when publication bias is present. In addition, subgroup analyses will be performed for 
further investigation. The above analyses will be performed using Stata 12.0.

Subgroup analysis: If enough studies are available, children and adults will be analyzed separately. When possible if enough data are available, we will measure males and females separately.

Sensitivity analysis: If enough studies are available, sensitivity analysis will be done.

Language: English.

Country(ies) involved: China.

Keywords: Sleep duration; Myopia; Glaucoma; Cataract; Age-related macular degeneration; Diabetic retinopathy.

Contributions of each author:

Author 1 - Miao Zhou - The author conducted the literature searches, extracted the data from published papers, carried out the statistical analysis, and drafted the manuscript.

Email: 2111210311@stu.pku.edu.cn

Author 2 - Jia-Yan Kai - The author conducted the literature searches and extracted the data from published papers.

Email: 1664150473@qq.com

Author 3 - Chen-Wei Pan - The author contributed to the conception and design of this paper.

Email: 15062317163@163.com 